\title{
Editorial: It's Already Been Five Years!
}

The old adage "Time flies when you're having fun" certainly strikes a resonance with the staff at Physical Review Applied! And before we knew it, the journal turned 5 years old, and now is well into its $6^{\text {th }}$ year. While still a child, Physical Review Applied is showing signs of growing into a robust and exciting member of the prestigious Physical Review family of journals.

This anniversary provides us with an opportunity to reflect on what has transpired over the last few years. I offer a few statistics that indicate the success of Physical Review Applied, and how it has become positioned as the premier journal dedicated to the diverse topics of applied physics:

- Journal Impact factor: 4.532

- Projected number of published papers in 2019: 900

- Compound annual growth rate of the number of papers published since inception: Approximately $30 \%$.

- Number of review papers published: 8

We are indeed grateful to our authors, reviewers, readers, and dedicated APS staff for this remarkable early success.

In addition, we published a unique collection of 10 papers over a period of nine months. This is the so-called "Millie Collection" honoring the extraordinary career and accomplishments of Mildred Dresselhaus, who was one of the inaugural Editorial Board Members of Physical Review Applied.

But the future looks even brighter than our past. For example, we have just launched a new collection of invited papers on "Two Dimensional Materials and Devices". This is an extremely timely and exciting topic, and we have solicited papers from authors whose work has made transformational changes to the field of limited dimensional systems. Assembly of this collection breaks new ground in an entirely different dimension as well. It has been launched as a joint venture between Physical Review Applied and Physical Review Materials. Acceptance of all papers in the collection are expected to be agreed upon by both journals, and cross-listed between them as a means to reach the broadest possible audience. We believe that partnering in this fashion provides greater value to everyone in the community, and indeed is itself a reflection of the interdisciplinary nature of some of the best science pursued today.

Finally, it is important to note that such rapid growth brings opportunities as well as strains to the system. We have increased our editorial staff during these first 5 years to keep up with the growth in submissions, but, given the large and growing volume of published papers, we can no longer continue to offer "teasers" that briefly provide highlights for every paper. Nevertheless, we recognize the value of these short summaries, and so we will still publish them for every Editors' Suggestion, Letter, and Review Article.

As Lead Editor of Physical Review Applied, I want to take this opportunity to once more thank all of you for your support of and confidence in our journal. It is because of this that the first $5+$ years have been so successful. But we know that to make the next 5 years even more successful, we need to earn that confidence every day. I speak for the entire staff at APS when I say that service to the physics community is without exception our primary goal. I look forward to the years ahead and, with your help, the growing stature of this journal.

Published 2 January 2020

Steve Forrest

Editor

DOI: 10.1103/PhysRevApplied.13.010001 